\title{
Supporting Information \\ Triboelectric Nanogenerator for Ocean Wave Graded Energy Harvesting and Condition Monitoring
}

Yuhong $\mathrm{Xu}^{\dagger, *, \perp}$, Weixiong Yang ${ }^{\dagger, *}, \perp$ Xiaohui $\mathrm{Lu}^{\ddagger}, \perp$, Yanfei Yang ${ }^{\dagger}$, Jianping $\mathbf{L i}^{\S}$, Jianming Wen ${ }^{*, \$}$, Tinghai Cheng ${ }^{*, \dagger},+, \|$, Zhong Lin Wang $*,+, \|, \#$

${ }^{\dagger}$ Beijing Institute of Nanoenergy and Nanosystems, Chinese Academy of Sciences, Beijing 101400, China

* School of Mechatronic Engineering, Changchun University of Technology, Changchun, Jilin 130012, China

$\$$ The Institute of Precision Machinery and Smart Structure, Key Laboratory of Intelligent Operation and Maintenance Technology \&

Equipment for Urban Rail Transit of Zhejiang Province, College of Engineering, Zhejiang Normal University, Yinbin Street 688, Jinhua, 321004, China

"CUSTech Institute of Technology, Wenzhou, Zhejiang 325024, China

\# School of Materials Science and Engineering, Georgia Institute of Technology, Atlanta, Georgia 30332-0245, United States

(a)

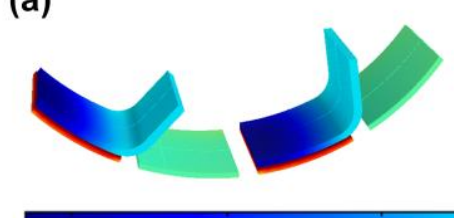

(b)

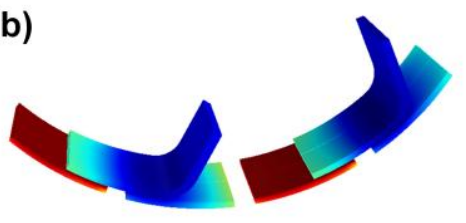

(c)

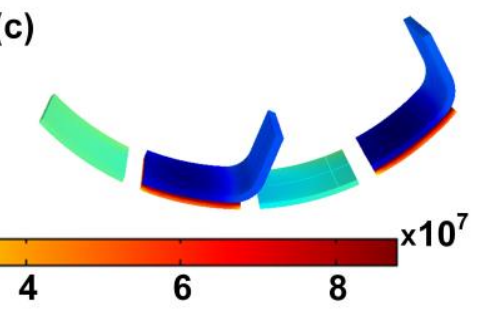

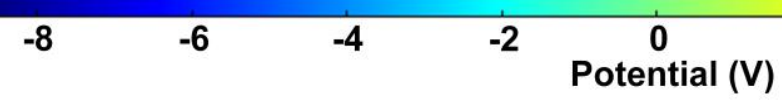

Figure S1. Simulations of the device in three states.

Because the overall structure of the two generation units is the same, under the same conditions, the open-circuit voltage $\left(V_{\mathrm{OC}}\right)$ and transfer charge $\left(Q_{\mathrm{SC}}\right)$ output by generation unit I and generation unit II are almost identical. With the increase of input frequency and amplitude, the $V_{\mathrm{OC}}$ and $Q_{\mathrm{SC}}$ of the GEH-TENG are almost unchanged, but their duration will increase. 


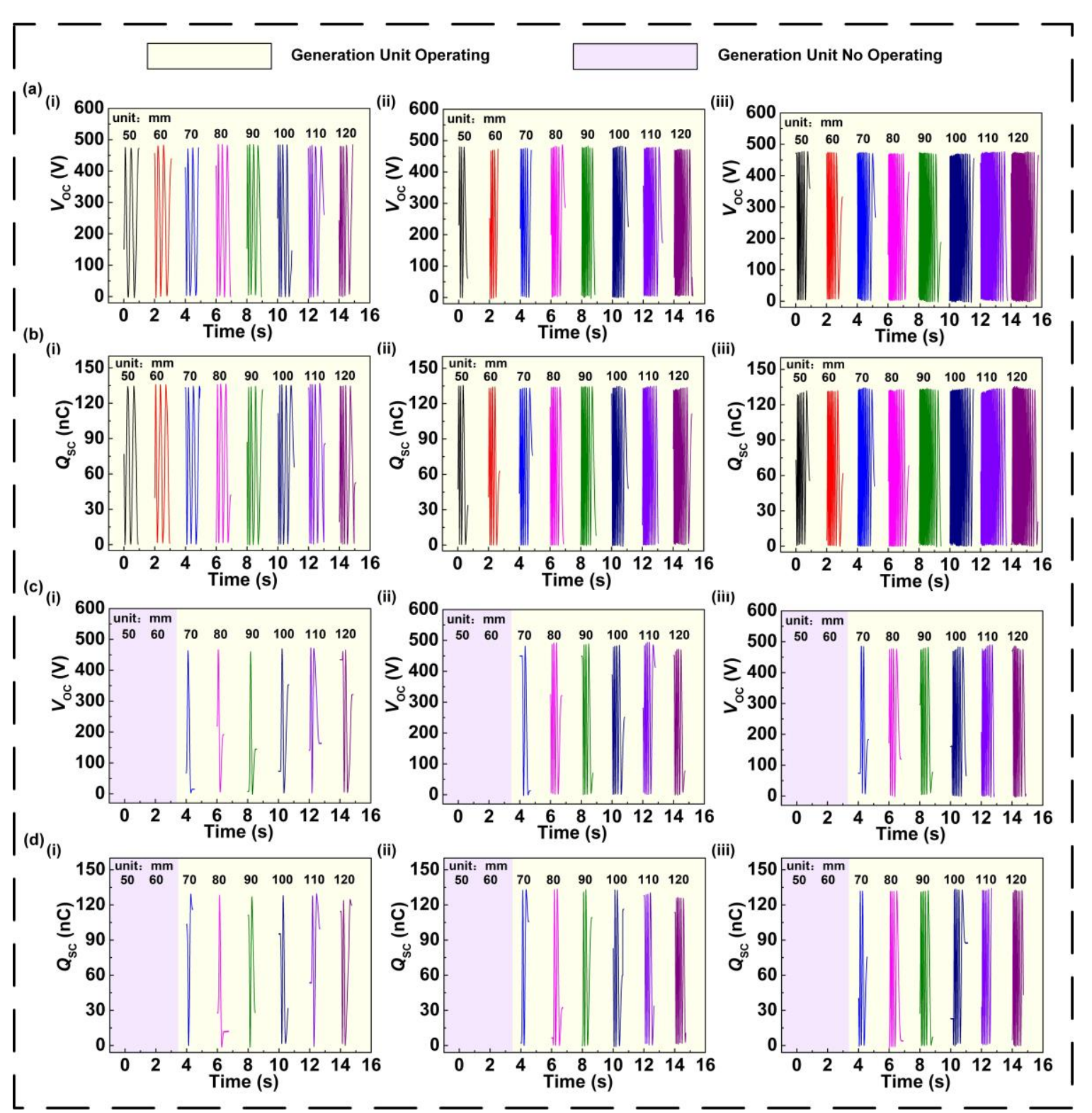

Figure S2. Open-circuit voltage $\overline{\text { and }} \overline{\text { transferred }} \overline{\text { charge comparison of the }} \overline{\mathrm{GEH}}-\overline{\mathrm{TEN}} \overline{\mathrm{und}} \overline{\mathrm{u}}$ different input amplitudes and input frequencies of $0.5 \mathrm{~Hz}, 1.0 \mathrm{~Hz}$, and $1.5 \mathrm{~Hz}$ with only $(\mathrm{a}, \mathrm{b})$ generation unit I and (c, d) generation unit II.

Under the same input amplitude, with the increase of mass, the maximum of load current through the external resistance of $50 \mathrm{M} \Omega$ also gradually increases (Figure S3a-c). When the external excitation frequency is $1.5 \mathrm{~Hz}$, the output $I_{\mathrm{DC}}$ signal with the counterweight condition of \#3 is shown in Figure S3d. With the increase of input amplitude, the peak of $I_{\mathrm{DC}}$ of the GEHTENG gradually increases. By amplifying the $I_{\mathrm{DC}}$ signals with amplitude of $110 \mathrm{~mm}$ and 120 $\mathrm{mm}$, can clearly see the working state changes of the two generation units of the GEH-TENG (Figure S3e-f). When an input amplitude of $120 \mathrm{~mm}$, the GEH-TENG can generate a $17 \mu \mathrm{A}$ $I_{\mathrm{DC}}$. 

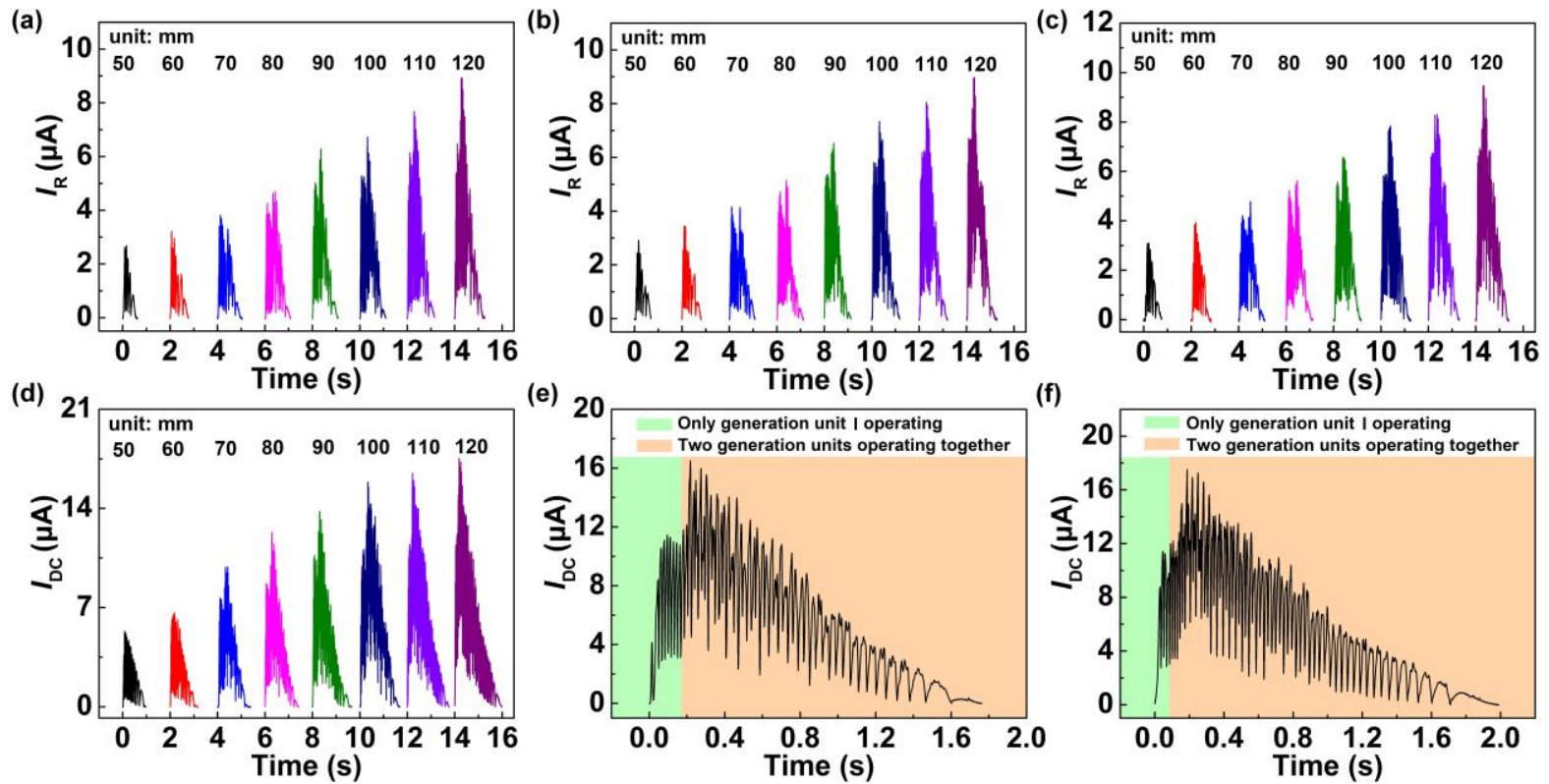

Figure S3. Two generation units are connected in parallel after rectification, comparison of output performance under different conditions. (a-c) Comparison of load current under three different mass plate number conditions of the GEH-TENG with different input amplitudes and input frequencies of $0.5 \mathrm{~Hz}, 1.0 \mathrm{~Hz}$, and $1.5 \mathrm{~Hz}$. (d) $I_{\mathrm{DC}}$ under counterweight condition of \#3 at $1.5 \mathrm{~Hz}$, (e-f) enlarged images of output signals with amplitudes of $110 \mathrm{~mm}$ and $120 \mathrm{~mm}$, respectively.

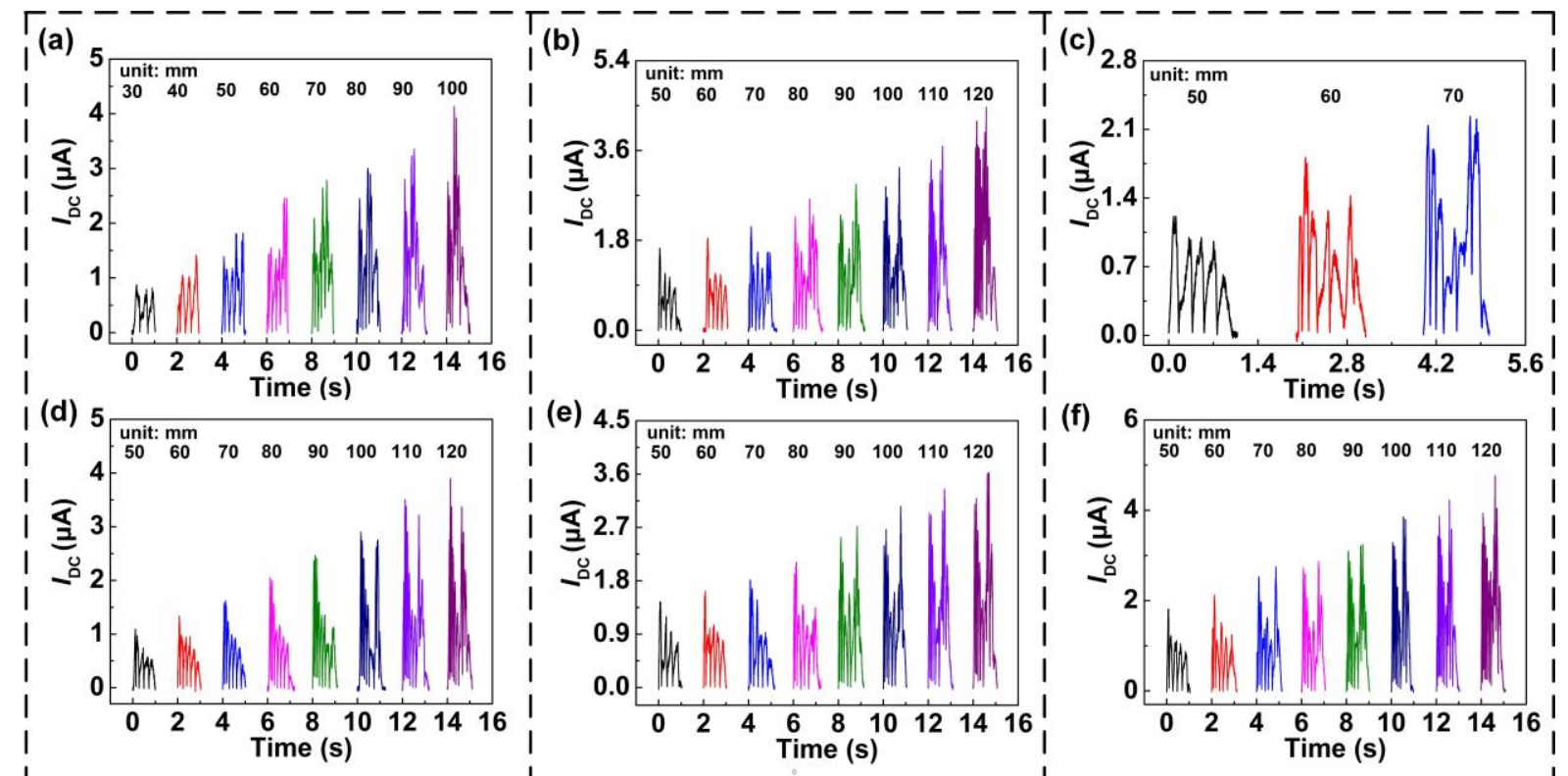

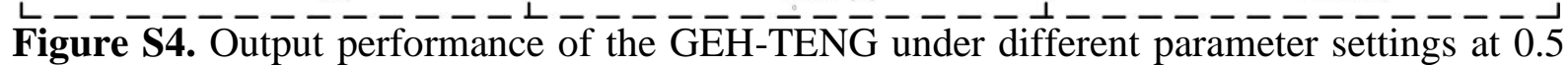
Hz: (a, d) length of the pendulum below the water surface is $60 \mathrm{~mm}$, length of the pendulum above the water surface is $80 \mathrm{~mm}$, and angle of the incomplete gears are $60^{\circ}$ and $100^{\circ} ;(\mathrm{b}, \mathrm{e})$ angle of the incomplete gear is $80^{\circ}$, length of the pendulum below the water surface is $60 \mathrm{~mm}$, and length of pendulum above the water surface is $90 \mathrm{~mm}$ and $100 \mathrm{~mm}$; (c, f) angle of the incomplete gear is $80^{\circ}$, length of the pendulum above the water surface is $80 \mathrm{~mm}$, and length of pendulum below the water surface is $20 \mathrm{~mm}$ and $40 \mathrm{~mm}$. 

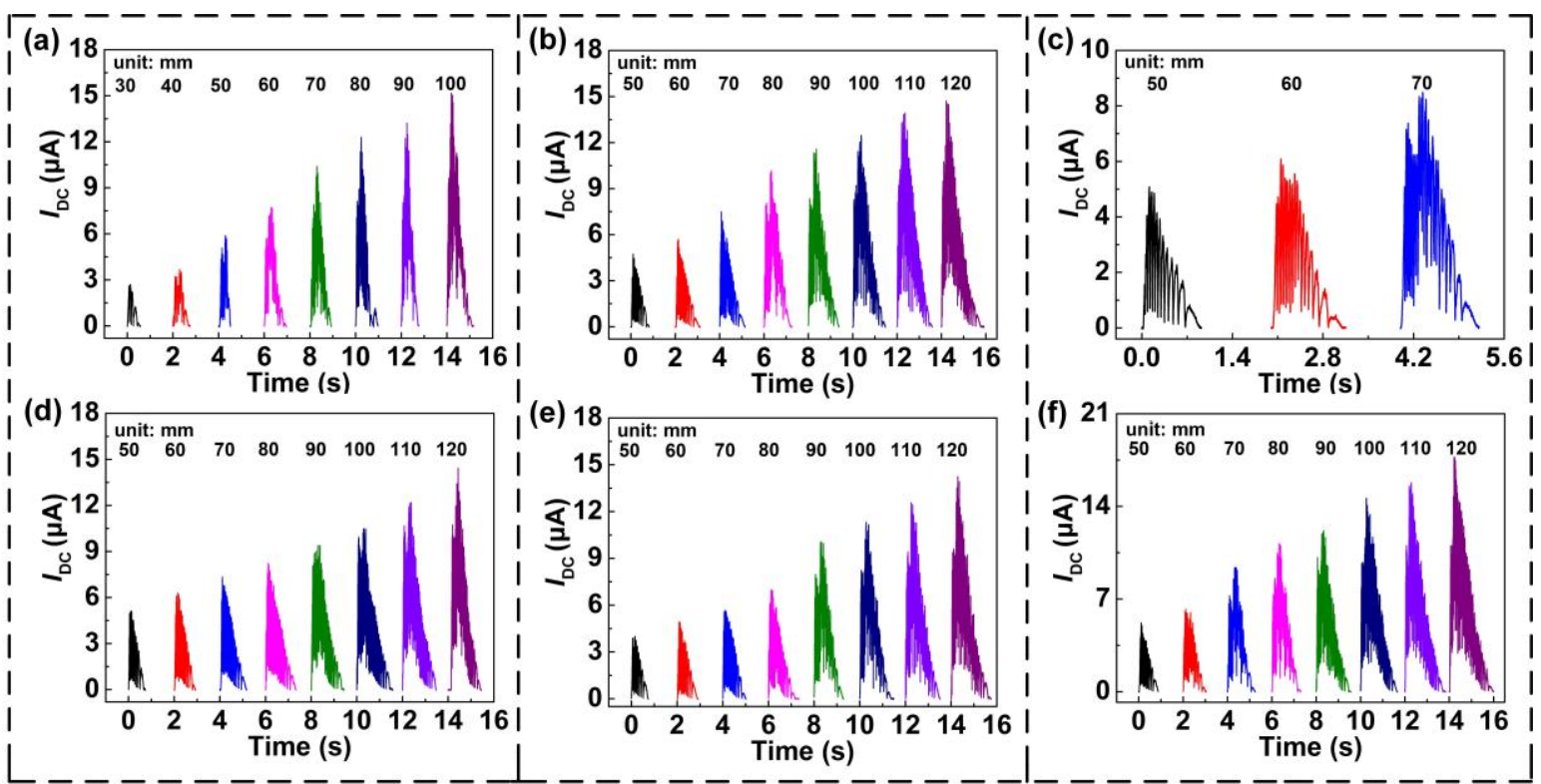

Figure S5. Output performance of the GEH-TENG under different parameter settings at 1.5 Hz: (a, d) length of the pendulum below the water surface is $60 \mathrm{~mm}$, length of the pendulum above the water surface is $80 \mathrm{~mm}$, and angle of the incomplete gears are $60^{\circ}$ and $100^{\circ} ;(\mathrm{b}, \mathrm{e})$ angle of the incomplete gear is $80^{\circ}$, length of the pendulum below the water surface is $60 \mathrm{~mm}$, and length of pendulum above the water surface is $90 \mathrm{~mm}$ and $100 \mathrm{~mm}$; (c, f) angle of the incomplete gear is $80^{\circ}$, length of the pendulum above the water surface is $80 \mathrm{~mm}$, and length of pendulum below the water surface is $20 \mathrm{~mm}$ and $40 \mathrm{~mm}$.
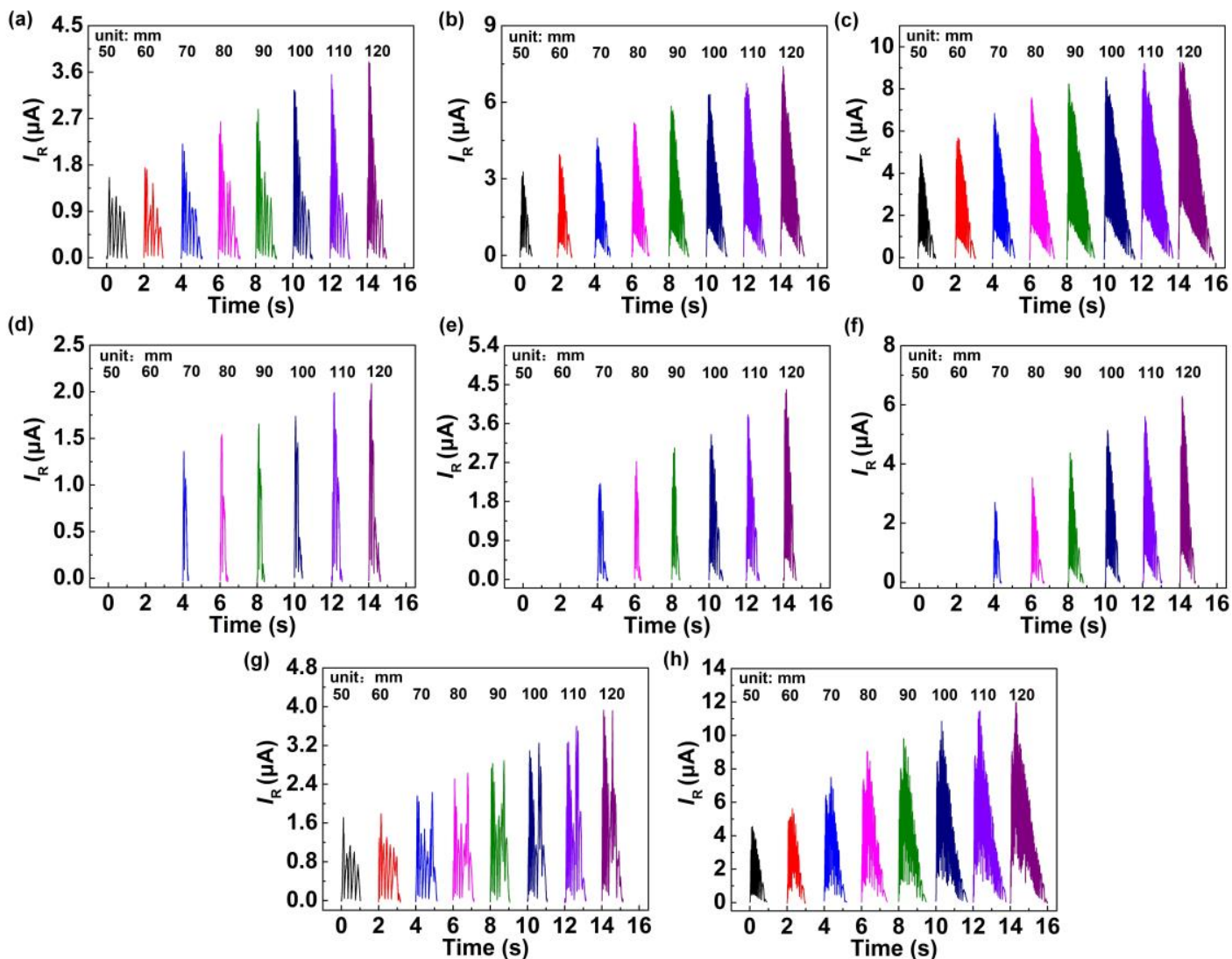

Figure S6. Load current comparison of the GEH-TENG with different input amplitudes: $(\mathrm{a}-\mathrm{c})$ only the generation unit I operates at input frequencies of $0.5 \mathrm{~Hz}, 1.0 \mathrm{~Hz}$, and $1.5 \mathrm{~Hz}$; (d-f) only the generation unit II operates at input frequencies of $0.5 \mathrm{~Hz}, 1.0 \mathrm{~Hz}$, and $1.5 \mathrm{~Hz} ;(\mathrm{g}, \mathrm{h})$ two generations operation together at input frequencies of $0.5 \mathrm{~Hz}$ and $1.5 \mathrm{~Hz}$. 
At the frequency is $0.5 \mathrm{~Hz}$ and $1.5 \mathrm{~Hz}$, the law of the GEH-TENG output energy is the same under different conditions. When the frequency is $1.5 \mathrm{~Hz}$ and the input amplitude reaches $120 \mathrm{~mm}$, the GEH-TENG with double generation units can generate $1.5 \mathrm{~mJ}$ of energy.

(a)

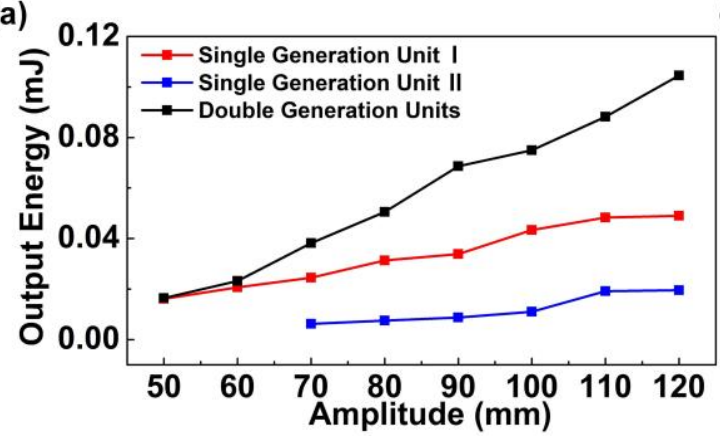

(b)

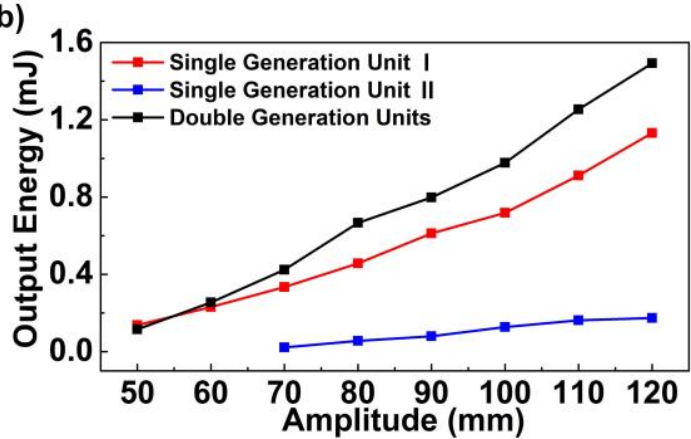

Figure S7. Comparison of output energy of the GEH-TENG under single and double generation units at different frequencies: (a) $0.5 \mathrm{~Hz}$, (b) $1.5 \mathrm{~Hz}$.

The calculation results show that the GEH-TENG, which under only generation unit I operation, reaches the peak power of $1.75 \mathrm{~mW}$ when the external resistance is $50 \mathrm{M} \Omega$ (Figure S8a). Under the same conditions, the GEH-TENG with only generation unit II operation, reaches the peak power of $1.33 \mathrm{~mW}$ (Figure S8b). The GEH-TENG with double generation unit operation, reaches the peak power of $4.63 \mathrm{~mW}$ (Figure S8c).
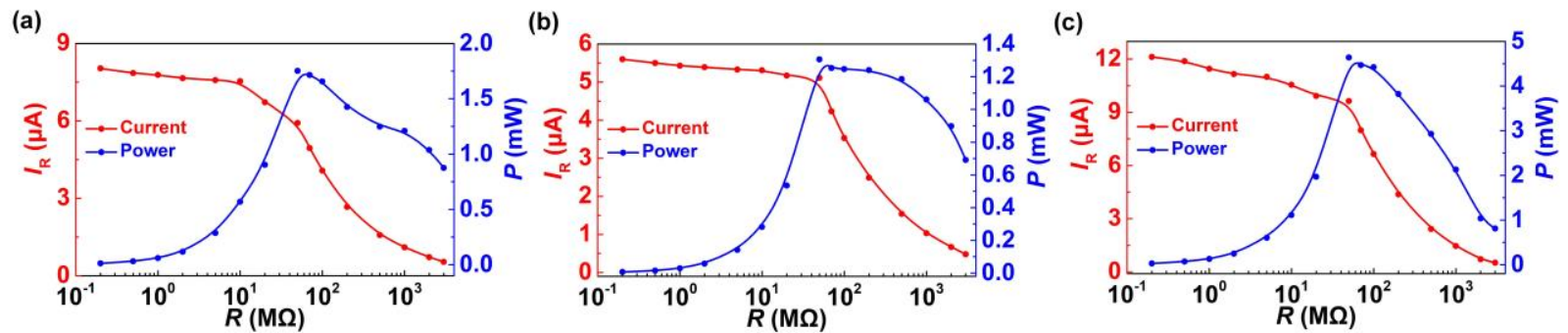

Figure S8. Output current and instantaneous output power of the GEH-TENG at different load resistances under (a) single generation unit I operation, (b) single generation unit II operation, and (c) double generation units operation.

The corresponding mechanical stability experiments are carried out on the same size generation unit set, to verify the influence of mechanical wear of FEP films and copper materials on the overall mechanical stability under long-term operation (Figure S9).

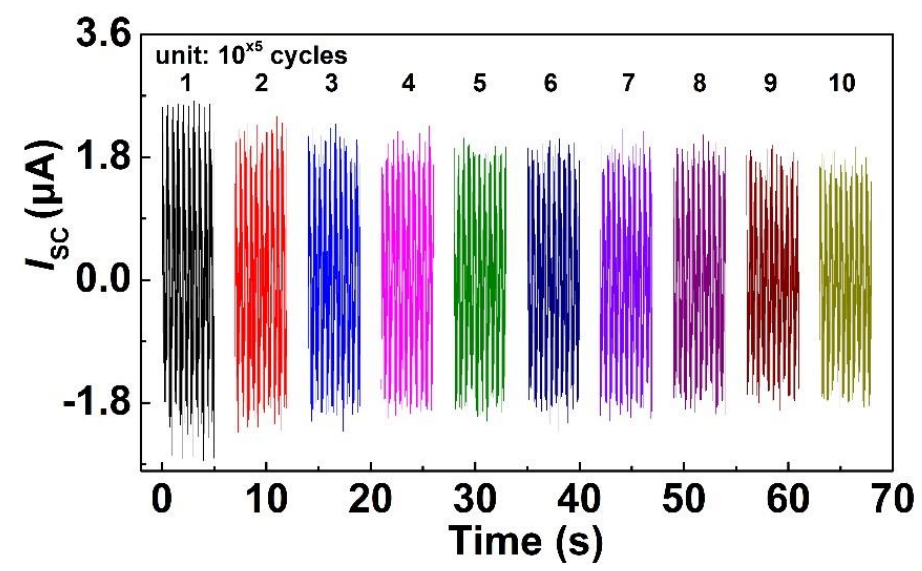

Figure S9. Performance change of generation unit during continuous operation for one million cycles at $60 \mathrm{rpm}$.

With the rotating machine as the excitation source, the generator unit continuously runs for one million cycles under the excitation condition of $60 \mathrm{rpm}$. After running for one million 
cycles, the output current is attenuated by $25 \%$, but the generation unit can still provide continuous current output.

To verify the durability of the GEH-TENG in the actual wave environment, the wave environment by using a pool and wave-making board is simulated. In the experiment, to avoid the influence of water waves on the output performance, the GEH-TENG is placed in a wellsealed acrylic box and placed on the water surface.

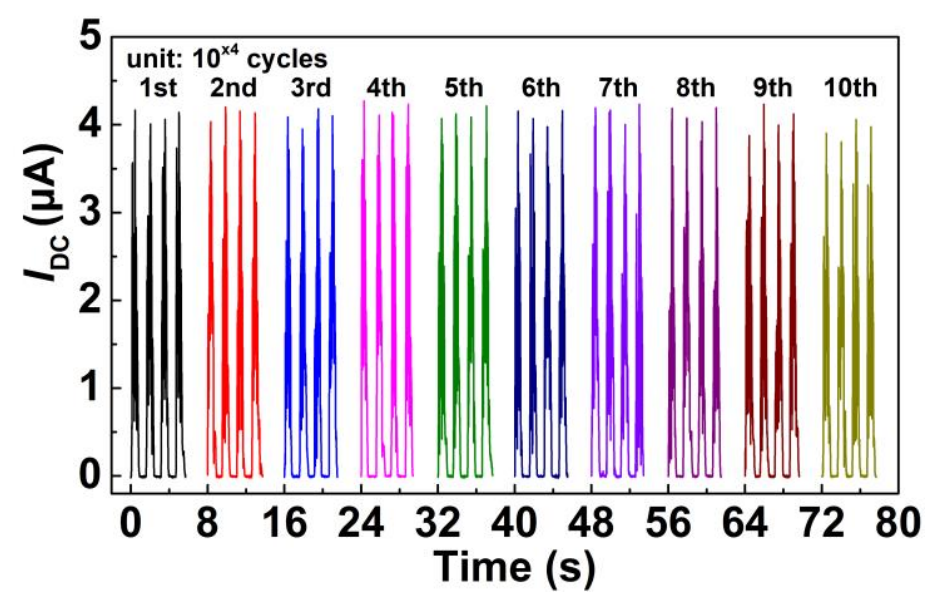

Figure S10. Output performance of the GEH-TENG in 100,000 cycles driven by waves.

In the experiment, the angle of the incomplete gear is set to $60^{\circ}$, length of the pendulum above the wave surface is set to $100 \mathrm{~mm}$, respectively. Under the wave excitation condition with an input frequency of $1.0 \mathrm{~Hz}$ and amplitude of $60 \mathrm{~mm}$, the GEH-TENG has continuously operated for 100,000 cycles (Figure S10). Its output performance has almost no attenuation, so the GEH-TENG has satisfactory durability. 